Portland State University

PDXScholar

7-1-2008

\title{
Creation of Nanopatterns by Local Protonation of P4VP via Dip Pen Nanolithography
}

\author{
Carsten Maedler \\ Portland State University \\ S. Chada \\ Portland State University \\ Xiquan Cui \\ Portland State University \\ M. Taylor \\ Portland State University \\ Mingdi Yan \\ Portland State University
}

See next page for additional authors

Follow this and additional works at: https://pdxscholar.library.pdx.edu/phy_fac

Part of the Physics Commons

Let us know how access to this document benefits you.

\section{Citation Details}

Maedler, C. C., Chada, S. S., Cui, X. X., Taylor, M. M., Yan, M. M., \& La Rosa, A. A. (2008). Creation of nanopatterns by local protonation of P4VP via dip pen nanolithography. Journal Of Applied Physics, 104(1), 014311.

This Article is brought to you for free and open access. It has been accepted for inclusion in Physics Faculty Publications and Presentations by an authorized administrator of PDXScholar. Please contact us if we can make this document more accessible: pdxscholar@pdx.edu. 
Authors

Carsten Maedler, S. Chada, Xiquan Cui, M. Taylor, Mingdi Yan, and Andres H. La Rosa 


\title{
Creation of nanopatterns by local protonation of P4VP via dip pen nanolithography
}

\author{
C. Maedler, ${ }^{1}$ S. Chada ${ }^{2}$ X. Cui, ${ }^{1}$ M. Taylor, ${ }^{2}$ M. Yan, ${ }^{2, a)}$ and A. La Rosa ${ }^{1, b)}$ \\ ${ }^{1}$ Department of Physics, Portland State University, Portland, Oregon 97201, USA \\ ${ }^{2}$ Department of Chemistry, Portland State University, Portland, Oregon 97201, USA
}

(Received 11 February 2008; accepted 3 May 2008; published online 11 July 2008)

The elastic mechanical response of a poly(4-vinylpyridine) film is exploited to create nanostructures under ambient conditions via dip pen nanolithography. Using a $p \mathrm{H} 4$ phosphate buffer solution as the "ink," a series of experimental results indicates that the nanometer-sized structures are due to local swelling of the film's pyridyl groups upon their protonation with the hydronium ions delivered by the tip. Control over the structures' height is gained by properly selecting the writing velocities or the dwell time, respectively. (c) 2008 American Institute of Physics. [DOI: 10.1063/1.2953090]

\section{INTRODUCTION}

Dip pen nanolithography (DPN) is a direct write chemical patterning technique ${ }^{1-4}$ that uses an atomic force microscope (AFM) tip as a "nanopen" to anchor "ink" molecules onto a target substrate surface. In many cases, the medium for this transport is a water meniscus that forms between the tip and the sample's surface. ${ }^{5,6}$ DPN has been applied to create a variety of micron- and nanosized patterns composed of small molecules, ${ }^{1}$ conducting polymers, ${ }^{7,8}$ biological macromolecules, ${ }^{9,10}$ polymer single crystals ${ }^{11} p \mathrm{H}$-sensitive block copolymers, ${ }^{12}$ inorganic materials, ${ }^{13,14}$ and single particles ${ }^{15}$ on metals, semiconductors, and monolayer functionalized surfaces. Generally, in order to create stable nanostructures, the ink molecules must be able to anchor themselves to the substrate via chemisorption or other electromagnetic interactions.

In contrast to the DPN anchoring-molecule approach for creating nanostructures, this manuscript describes a DPN method that instead exploits the swelling mechanical response of the substrate. Indeed, a series of experimental tests (described below) provides evidence that an AFM tip, when coated with phosphate buffer solution of $p \mathrm{H} 4$ and placed in contact with the substrate, delivers protons into a poly(4vinylpyridine) ( $\mathrm{P} 4 \mathrm{VP}$ ) polymer film causing the latter to swell. These experiments constitute a nanometer-scale version of previously reported swelling of UV-crosslinked P4VP films under acidic stimulation but in a macroscale setup. ${ }^{16}$ In that experiment, measurements performed before and after soaking P4VP films in a $p \mathrm{H} 4$ buffer solution indicated a $20 \%$ increase in the films' thickness (measured by ellipsometry of the dried films), while a shift in the (IR-spectra) frequency band associated with the pyridyl ring-stretching modes provided a signature of the protonation.

The increase in film thickness is due to the protonation of pyridyl groups by the added acidic component to form pyridinium ions whose mutual repulsions cause the film to expand; this minimizes repulsion around the charged centers (Fig. 1). Triggering the polymer swelling mechanism via

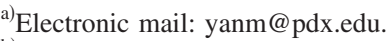

b)Electronic mail: andres@pdx.edu.
}

DPN, as described in this manuscript, establishes a way to create nanostructures, which is different from other nanolithography methods.

\section{EXPERIMENTAL DETAILS}

Materials. P4VP (molecular weight approximately 160 000, Aldrich), $n$-butanol (Fisher Scientific) were used as received. Sodium phosphate monobasic and sodium phosphate anhydrous dibasic were obtained from Mallinckrodt Chemical Works.

Preparation of polymer thin films. A $10 \mathrm{mg} / \mathrm{ml}$ solution of P4VP was prepared in $n$-butanol. Silicon wafers with a native oxide thickness of $10 \AA$ were cut into $1 \times 1 \mathrm{~cm}$ square pieces, cleaned by sonicating in isopropyl alcohol for 15 min, and subsequently dried under a stream of nitrogen. Afterward, a P4VP solution was spin coated onto the wafers at $2000 \mathrm{rpm}$ for $60 \mathrm{~s}$, followed by irradiation using a $450 \mathrm{~W}$ medium-pressure mercury lamp for $5 \mathrm{~min}$ (the latter included $\sim 2$ min warm-up time for the lamp to reach its maximal intensity). The $5.1 \mathrm{~mW} / \mathrm{cm}^{2}$ intensity of the lamp was measured using a sensor of peak sensitivity at a wavelength

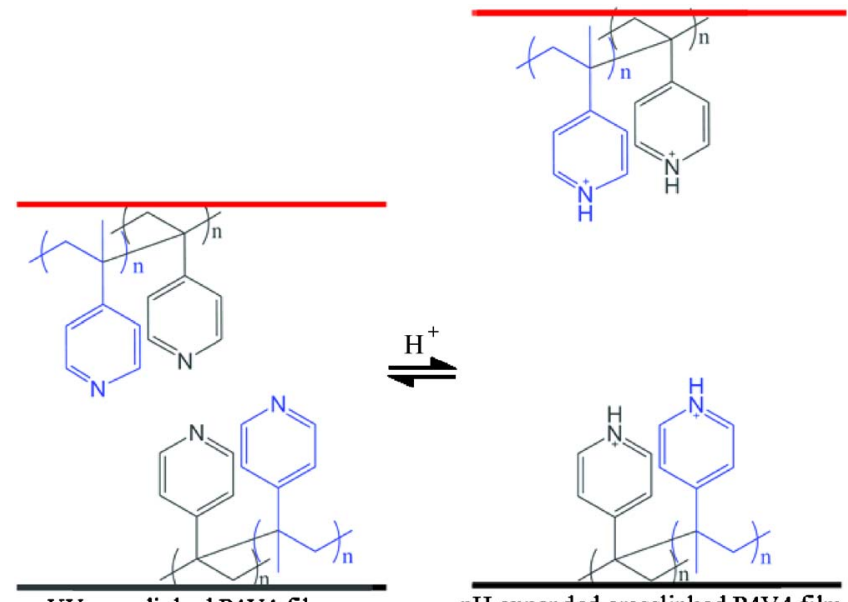

UV crosslinked P4V4 film

FIG. 1. (Color online) Proposed mechanism of film's pyridyl groups swelling upon their protonation with hydronium ions. The film expands to minimize repulsion around the charged centers. 


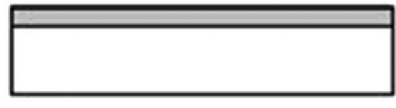

Spin-coat P4VP onSiwafer

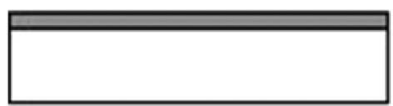

Crosslink P4WP under UV

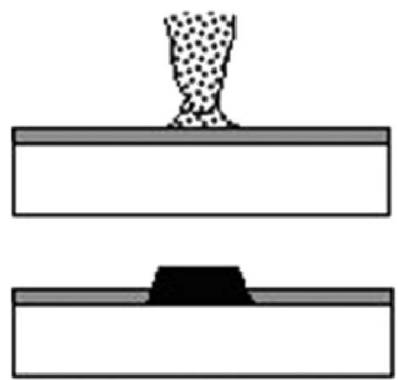

\section{DPN with $P B$}

buffer

The written

area swelled

FIG. 2. Procedure for implementing spatially localized protonation and swelling of a P4VP polymer film via DPN.

of $254 \mathrm{~nm}$. The irradiated films were then soaked in $n$-butanol for $24 \mathrm{~h}$ to remove the unbound polymer. The thickness of the film was measured with a Gaertner ellipsometer (model L116A, with a $2 \mathrm{~mW} \mathrm{He} / \mathrm{Ne}$ laser at an incident angle of $70^{\circ}$ ), using as refractive indices 1.465 for $\mathrm{SiO}_{2}$ and 1.581 for P4VP in the final estimations. The average of measurements at three different locations gave a typical thickness of $37 \pm 2 \mathrm{~nm}$.

Preparation of the AFM tip. Phosphate buffer solution $(0.1 M)$ of $p \mathrm{H} 4$ was prepared using $\mathrm{NaH}_{2} \mathrm{PO}_{4}$ and $\mathrm{Na}_{2} \mathrm{HPO}_{4}$. AFM tips ( $\mathrm{Si}, \mathrm{NSC} 15$, Mikromasch, $k=40 \mathrm{~N} / \mathrm{m}$ ) were coated by simply soaking the probe into the buffer solution for $1 \mathrm{~min}$, and then allowing them to dry in air for $10 \mathrm{~min}$. Subsequently, the tip was mounted on the head stage of a multimode scanning probe microscope (Nanoscope III, Veeco). In a given session, the same tip was used for all the intended test experiments but cleaned and coated anew before each trial. In order to verify that the starting substrate topography was smooth enough, all the samples were first scanned (with the buffer coated tip) at a relatively fast scan rate $(2 \mathrm{~Hz}$ or $5 \mu \mathrm{m} / \mathrm{s}$ ) and in tapping mode (TM). The use of high scanning rates prevented transferring buffer molecules to the surface. Subsequently, line structures were fabricated using slower scanning rates (as detailed below). A step by step process of the polymer pattern formation is outlined in Fig. 2.

A systematic procedure list was outlined for creating nanostructures under different fabrication conditions, as well as for testing hypothesis about the patterning working mechanism. (The interpretation of the results is provided in Sec. III below.)

(i) Scanning along crossed lines on a P4VP film with a coated probe. Operating the AFM in contact mode $(\mathrm{CM})$, a line scan was performed using the following parameters: scanning speed of $100 \mathrm{~nm} / \mathrm{s}$ ( or $0.04 \mathrm{~Hz}$ ), contact force of $5 \mu \mathrm{N}$, scanning length of $2.5 \mu \mathrm{m}$, and scan angle of $45^{\circ}$. The formation of the line pillar was subsequently verified by imaging the sample in
TM. A second line scan was performed afterward under the same parameters and conditions, except using this time a scan angle of $135^{\circ}$.

(ii) Writing on a P4VP film with a coated probe and under different contact forces. Three different line scans were performed using contact forces of $1 \mu \mathrm{N}, 2 \mu \mathrm{N}$, and $3 \mu \mathrm{N}$, respectively; all of them at the same scanning speed of $80 \mathrm{~nm} / \mathrm{s}$ and scanning length of $2 \mu \mathrm{m}$.

(iii) Writing on a P4VP film using an uncoated tip. For comparison purposes, a P4VP film was scanned with an uncoated tip in order to find out whether or not pillar polymer structures could result. A line scan was performed at a contact force of $3 \mu \mathrm{N}$ and a scanning speed of $140 \mathrm{~nm} / \mathrm{s}$.

(iv) Scanning on an alternative polystyrene film using a coated tip. A polystyrene film was selected because a macroscopic scale version of this experiment verified that this film does not swell when soaked into acidic buffer solutions. The polystyrene film was scanned with a coated tip in $\mathrm{CM}$ at a scanning speed of $80 \mathrm{~nm} / \mathrm{s}$. The parameters were similar to the ones used in case (ii) mentioned above, namely, a contact force of $2 \mu \mathrm{N}$ along a scanning length of $2 \mu \mathrm{m}$.

(v) Creating dot features using different dwell times. The tip was engaged and retracted at the same spot after three different dwell times in the following order: 2, 4 , and $6 \mathrm{~s}$ using the same contact force in each case.

(vi) Testing the stability of the created structures. Two images of the same sample region were taken $1 \mathrm{~h}$ apart.

\section{RESULTS AND DISCUSSION}

(i) Crossed lines patterned on a P4VP film. The first line scan in CM produced a structure of $150 \mathrm{~nm}$ in width, $5 \mu \mathrm{m}$ in length, and averaged $20 \mathrm{~nm}$ in height (Fig. 3 ); the height varies within the 16 and $24 \mathrm{~nm}$ range. The second line scan produced a line pattern oriented perpendicular to the first. Although the second line scan was implemented under similar conditions as the first, it resulted in a line pattern of only $5 \mathrm{~nm}$ in height. We believe that the difference in height was caused by the running out of the buffer molecules on the AFM tip while taking several images of the first line. Further scanning without renewal of the coating did not lead to the creation of any new structures. Although we obtained better results in subsequent experiments this might be a limiting factor for the creation of nanopatterns, which could be improved by the use of nanofountain probes. ${ }^{17}$

(ii) Patterning a P4VP film under different contact forces. Lines of increasing heights result when applying stronger contact forces (see image at the left side in Fig. 4) Contact forces of 1,2 , and $3 \mu \mathrm{N}$ produced lines of heights equal to 7,12 , and $25 \mathrm{~nm}$, respectively (the former was created first as to prevent that the eventual running out of the ink could compromise our conclusions in this test). The dependence of the height on the applied force shows that the number of delivered molecules increases with the contact force, 


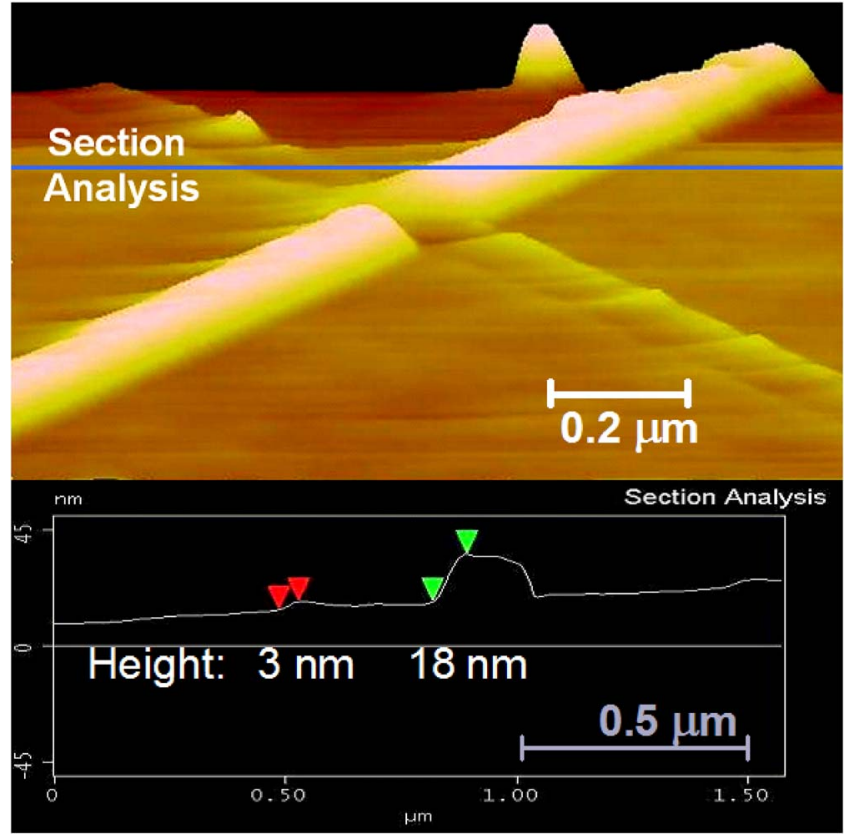

FIG. 3. (Color online) Crossed lines patterned on a P4VP film using a tip coated with a $p \mathrm{H} 4$ phosphate buffer solution.

which could be attributed to a greater effective tip area in contact with the polymer film or simply to a meniscus of wider diameter between the tip and substrate, thus leading to a more effective transfer of molecules. This result contrasts with what is found in the more typical (anchor based) DPN methods, where no dependence on the applied contact forces is observed. $^{4,18}$

(iii) Lack of pillar-type pattern formation when a P4VP polymer is scanned with an uncoated tip. When using a contact force similar to the strongest force in experiment (ii) only "scratches" were produced in the polymer film. The image at the center of Fig. 4 shows a dent $2 \mathrm{~nm}$ deep, accompanied by fairly low elevated features (apparently produced by the displaced material from the scratches) on the nearby sides. The structure of the line is obviously different from the ones
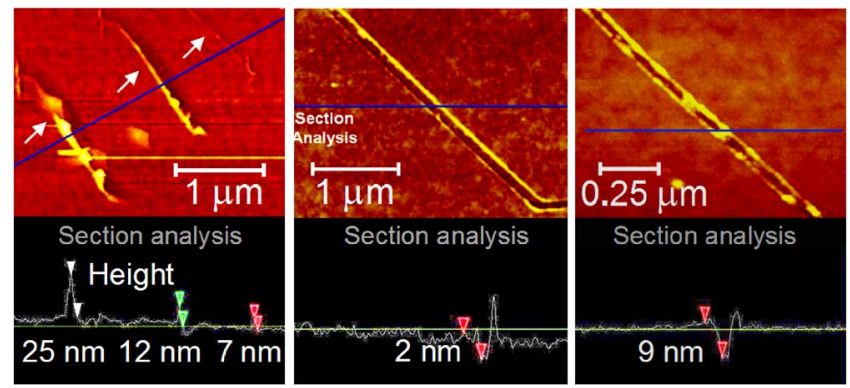

FIG. 4. (Color online) Left: Pillar-type patterns created on a P4VP film substrate using a coated tip under different contact forces: 1,2 , and $3 \mu \mathrm{N}$, from right to left, respectively. Center: A line scan on a P4VP film with an uncoated tip produces only scratches. The cross section line profile at the bottom suggests that there has been a removal of material, which accumulated at the right side of the line scratch. Right: A line scanning on a polystyrene film with a coated tip that causes only a scratch indent in the substrate.
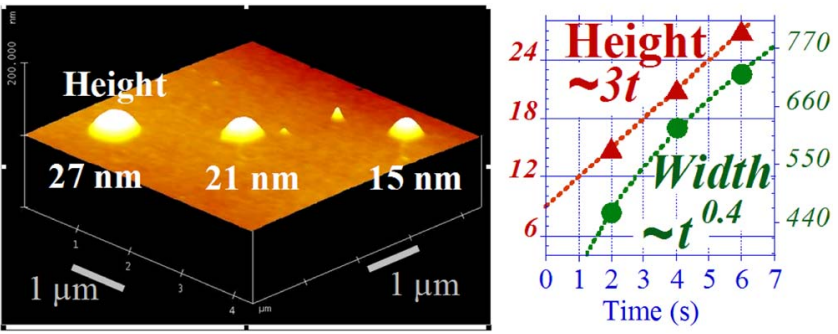

FIG. 5. (Color online) Left: Dots of controlled height fabricated using different dwell times. Right: Linear fitting to the dot's height vs time experimental values (filled triangles trace). The width, changing at a rate $t^{0.4}$, closely resembles a lateral diffusion process (filled circle trace). The vertical scales (left for the height; right for the width) are in nanometer units.

created with a coated tip, which supports our claim that the buffer solution is the cause for the elevated pillar lines created in experiments (i) and (ii).

(iv) Nonexistence of pillar-type pattern formation when a polystyrene film is scanned with an uncoated tip. A control experiment was carried out using a photocrosslinked polystyrene thin film. As shown in Fig. 4 (image on the right side) no elevated pillar line resulted from this procedure; rather a scratch with a depth of $9 \mathrm{~nm}$ is observed. This result is similar to the result obtained when writing on P4VP without buffer molecules and further supports the conclusion that the pillar lines in experiments (i) and (ii) are indeed produced by a polymer that swells under the action of protonation.

(v) Dot patterns of controlled height. "Dot" patterns of different heights are obtained when keeping the AFM tip in contact with the surface (without scanning) during different intervals of time (see image on the left side of Fig. 5). The dot corresponding to a dwell time of $2 \mathrm{~s}$ is $15 \mathrm{~nm}$ in height and $460 \mathrm{~nm}$ in width. For a dwell time of $4 \mathrm{~s}$ the dot is $21 \mathrm{~nm}$ in height and $620 \mathrm{~nm}$ in width. The dot produced at a dwell time of $6 \mathrm{~s}$ is $27 \mathrm{~nm}$ high and $720 \mathrm{~nm}$ wide. (The increasing size of the structures with the dwell time agrees with other similar tests-not shown here-including writing at different scanning speeds. Lines produced at a speed of $100 \mathrm{~nm} / \mathrm{s}$ were consistently taller than the ones produced at a speed of $200 \mathrm{~nm} / \mathrm{s}$, suggesting that by moving the tip slower more acid molecules are transferred to the polymer.) Figure 5 also shows a plot of the dots' width as a function of time, with a fitting curve proportional to $t^{0.4}$, which is indicative of a diffusion-type phenomenon. The latter may constitute the limiting factor for obtaining smaller features. This result suggests a mechanism model of the pattern formation: acid molecules from the tip are transferred to the sample substrate by laterally diffusing along the surface and then (or simultaneously) penetrating the substrate.

(vi) Stable patterns. Figure 6 shows images of the same region taken with $1 \mathrm{~h}$ time interval difference. Notice that the patterns remained unaltered, which indicates that the structures are not constituted by a volatile material, for example, water from the meniscus 


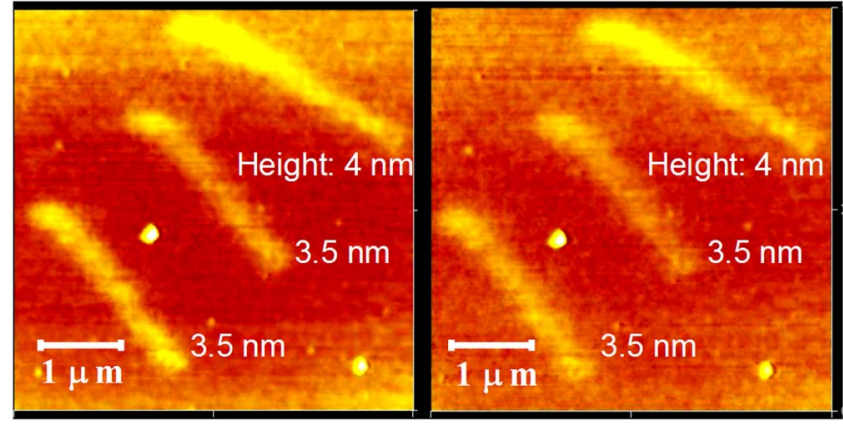

FIG. 6. (Color online) AFM images of the same sample region taken $1 \mathrm{~h}$ apart.

formed between the tip and substrate. ${ }^{19,20}$ This result also reveals an absence of potential postdiffusion that acid molecules may had undergone if they had been left present on the pillar patterns, since all the geometrical dimensions of the standing features remained significantly unaltered. The absence of pillar patterns when writing on other polymer samples that do not swell [section (iv) above] also makes the presence of acid molecules responsible for the patterns very unlikely.

As in other DPN experiments, the uncertainty in pattern formation is always a problem because of the complex processes of DPN, including, for example, the formation of the water meniscus condensation and the diffusion of the ink molecules onto the substrate..$^{21}$ Humidity, temperature, and morphology of both tip and substrate can all influence the result of DPN.

\section{CONCLUSION}

We have demonstrated the creation of polymer nanostructures and provided evidence that the driving mechanism is the local protonation of the UV-crosslinked P4VP films with molecules from a $p \mathrm{H} 4$ buffer solution delivered by an AFM tip. The height of the structures can be controlled by changing the contact force (a distinct feature that differentiates this method from the more common DPN anchoring approach) or by the scanning rate of the AFM tip. This capability to control the mechanical response of materials at the nanometer scale offers potential technological applications. In the area of biotechnology, for example, it can be employed to construct valve gates in nanofluidic devices ${ }^{22}$ or pillar arrays to sort out molecules by their sizes. ${ }^{23}$ Because the swelling of $\mathrm{P} 4 \mathrm{VP}$ is a reversible process, this technique may also find uses in erasable nanostorage devices. Additional studies about the dependence of the protonation on humidity and the reversible process are underway.

\section{ACKNOWLEDGMENTS}

M. Y. acknowledges the financial support from the donors of the Petroleum Research Fund, administered by the American Chemical Society (PRF \#43469 AC7), and NIH through an AREA Award GM066279. A. L. R. acknowledges support from the USA Air Force Office of Scientific Research through Grant No. FA9550-07-1-0511, and by the Oregon Nanoscience and Microtechnologies Institute through Grant No. N00014-07-1-0457.

${ }^{1}$ R. D. Piner, J. Zhu, F. Xu, S. Hong, and C. A. Mirkin, Science 283, 661 (1999).

${ }^{2}$ S. Hong, J. Zhu, and C. A. Mirkin, Langmuir 15, 7897 (1999).

${ }^{3}$ S. Hong, J. Zhu, and C. A. Mirkin, Science 286, 523 (1999).

${ }^{4}$ S. Hong and C. A. Mirkin, Science 288, 1808 (2000).

${ }^{5}$ R. D. Piner and C. A. Mirkin, Langmuir 13, 6864 (1997).

${ }^{6}$ D. L. Malotky and M. K. Chaudhury, Langmuir 17, 7823 (2001).

${ }^{7}$ B. W. Maynor, S. F. Filocamo, M. W. Grinstaff, and J. Liu, J. Am. Chem. Soc. 124, 522 (2002).

${ }^{8}$ A. Noy, A. E. Miller, J. E. Klare, B. L. Weeks, B. W. Woods, and J. J. DeYoreo, Nano Lett. 2, 109 (2002).

${ }^{9}$ D. L. Wilson, R. Martin, S. Hong, M. Cronin-Golomb, C. A. Mirkin, and D. L. Kaplan, Proc. Natl. Acad. Sci. U.S.A. 98, 13660 (2001).

${ }^{10}$ L. M. Demers, D. S. Ginger, S.-J. Park, Z. Li, S.-W. Chung, and C. A. Mirkin, Science 296, 1836 (2002).

${ }^{11}$ X. Liu, Y. Zhang, D. K. Goswami, J. S. Okasinski, K. Salaita, P. Sun, M. J. Bedzyk, and C. A. Mirkin, Science 307, 1763 (2005).

${ }^{12}$ D. J. Díaz, J. E. Hudson, G. D. Storrier, H. Abruña, N. Sundararajan, and C. K. Ober, Langmuir 17, 5932 (2001).

${ }^{13}$ X. Liu, L. Fu, S. Hong, V. P. Dravid, and C. A. Mirkin, Adv. Mater. (Weinheim, Ger.) 14, 231 (2002).

${ }^{14}$ M. Su, X. Liu, S. Y. Li, V. P. Dravid, and C. A. Mirkin, J. Am. Chem. Soc. 124, 1560 (2002).

${ }^{15}$ Y. Wang, Y. Zhang, B. Li, J. Lü, and J. Hu, Appl. Phys. Lett. 90, 133102 (2007).

${ }^{16}$ B. Harnish, J. T. Robinson, Z. Pei, O. Ramstrom, and M. Yan, Chem. Mater. 17, 4092 (2005).

${ }^{17}$ K.-H. Kim, N. Moldovan, and H. D. Espinosa, Small 1, 632 (2005).

${ }^{18}$ B. L. Weeks, A. Noy, A. E. Miller, and J. J. De Yoreo, Phys. Rev. Lett. 88, 255505 (2002).

${ }^{19}$ R. D. Piner and C. A. Mirkin, Langmuir 13, 6864 (1997).

${ }^{20}$ Reference 19 reports the deposition of water molecules from the (tipsample) meniscus onto the substrate. However, the thickness of these films, under various ambient conditions, is of the order of $0.1 \mathrm{~nm}$, quite small compared to the film thickness reported in this manuscript.

${ }^{21}$ J. Jang, S. Hong, G. C. Schatz, and M. A. Ratner, J. Chem. Phys. 115, 2721 (2001).

${ }^{22}$ S. R. Quake and A. Scherer, Science 290, 1536 (2000).

${ }^{23}$ L. R. Huang, E. C. Cox, R. H. Austin, and J. C. Sturm, Science 304, 987 (2004). 
Journal of Applied Physics is copyrighted by the American Institute of Physics (AIP). Redistribution of journal material is subject to the AIP online journal license and/or AIP copyright. For more information, see http://ojps.aip.org/japo/japcr/jsp 